\title{
INVITED PAPER \\ ISH 2022 KYOTO: A good trend for the on -site meeting
}

\author{
HIROSHI ITOH M.D. PH.D \\ President, ISH2022, Vice President, ISH \\ Professor and Chairman of Endocrinology, Metabolism and Nephrology, Keio University \\ School of Medicine, Tokyo Japan
}

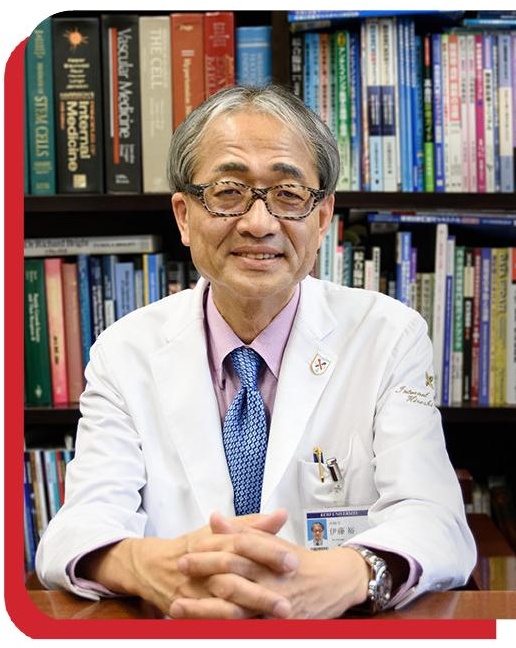

DOI: $10.30824 / 2112-11$

The $29^{\text {th }}$ Scientific Meeting of the International Society of Hypertension (ISH2022) will be held from October 12 to 16 in 2022 at Kyoto International Conference Center with the theme of "The Wisdom for Conquering Hypertension" (Figure 1). We will hold the meeting on-site in a hybrid format that will also provide online contents. The local organizing committee members are intensively preparing for the meeting hoping that hypertension researchers in the world will meet in person and share the scientific progress at Kyoto. Meanwhile, the utilization of online devices will be beneficial for participation of young researchers, students and colleagues in developing countries, for whom it has been difficult to visit Japan.

The number of patients with COVID-19 in Japan has decreased steadily since this September, and the number of newly infected persons is shifting between 100 and 200 people/day as of November 2021. The number per capita marks one of the lowest in the world. The isolation period for overseas travelers after entering Japan has been shortened, making it easier for overseas travelers to enter Japan. We believe that Japanese situation would be much more improved next year to welcome oversea participants to
Japan. October is one of the best months to visit Kyoto because of the mild climate: little rain, neither too hot, nor too cold. Kyoto is the ancient capital of Japan with a current population of 1.47 million, where many cultural heritage sites and traditional scenery can be seen (Figure 2). The atmosphere from ancient times blends into the vibrancy of a big city.

By holding a face to face gathering at the ISH2022 Kyoto, our friends and colleagues from all over the world will communicate in person and share the scientific progress on conquering hypertension. The number of participants is estimated to be at least 4500 people. We are planning to prepare enough grants: travel grants for 300 young participants, poster awards for developing countries, and excellent presentation awards. Support from various organizations has been dedicated, including the Ministry of Health, Labor and Welfare in Japan, and the Japan Medical Association. The Science Council of Japan, which is one of the leading organizations for scientific research promotion in Japan under direct control of the Cabinet Office, has officially decided to support ISH2022, showing their high interests in the study of Hypertension, and acknowledging the importance of

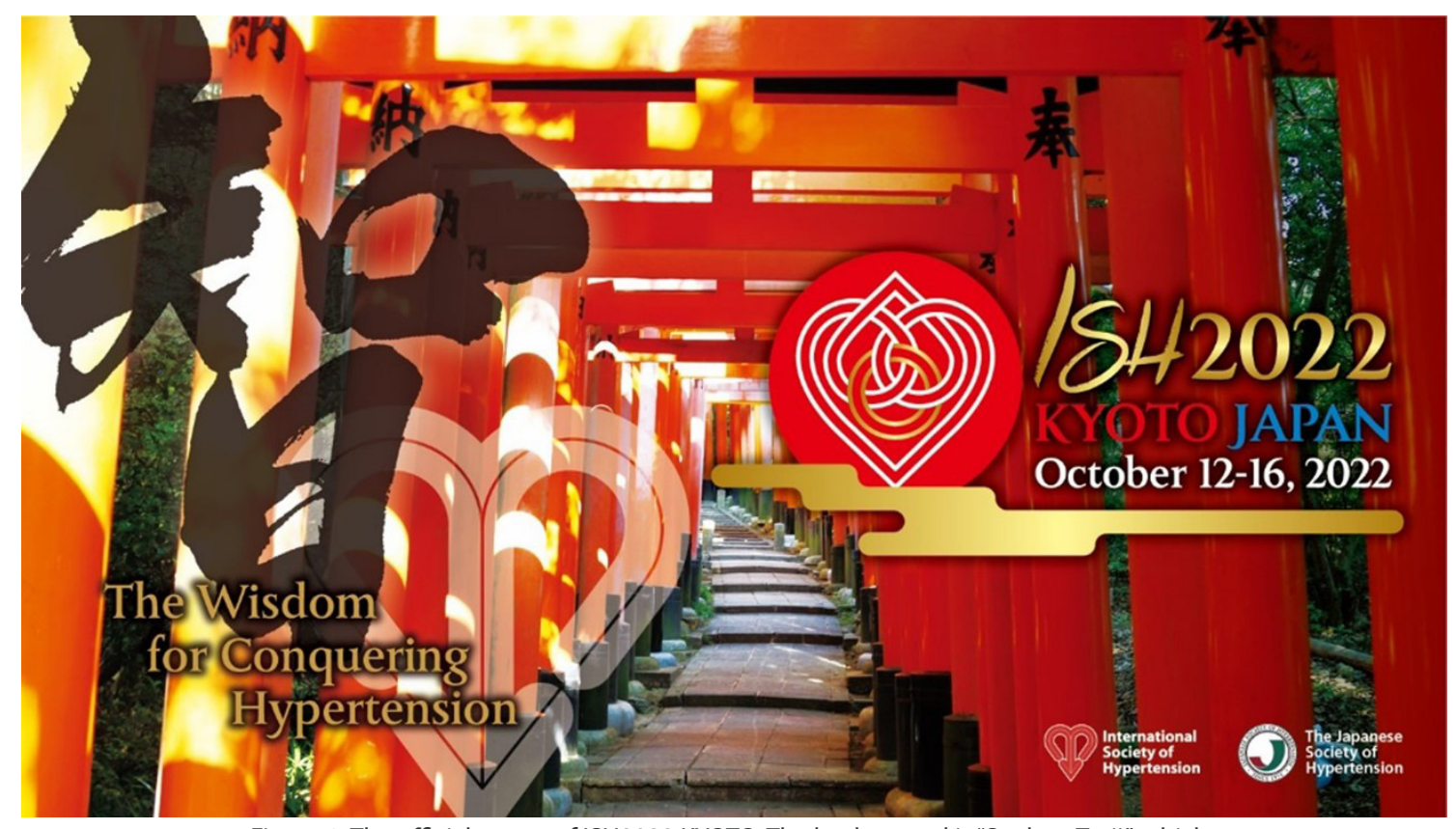

Figure 1. The official poster of ISH2022 KYOTO. The background is "Senbon Torii", which is a path with thousand wooden gates of Fushimi Inari shrine in Kyoto. 
it. To share the world's most advanced knowledge on hypertension research, outstanding intelligence from all over the world has been invited (Figure 3). As a speaker outside the field of hypertension research, Dr Hiroshi Ishiguro, who is an authority on robotics in the world, will give a lecture.

ISH2022 are focusing on the three main topics: Food (food and nutrition), Move (exercise and fitness) and Al (artificial intelligence and digital health), that are related to hypertension (Figure 4). The participants are expected to include not only academia and medicine but also members of various companies and local governments. The program committee members are intensively working to make ISH2022 innovative, impressive and informative for participants and has decided to cover the following categories. The symposiums will be organized by both the invited speakers of the state of art and those who are publicly recruited at the same session to seek the synergetic effects. In addition to general symposiums, programs such as TED style lectures, Debate and Case studies are included in the meeting.

Categories of the symposiums in ISH2022 KYOTO.

1. Hypertension for Sustainable Development Goals (SDGs) Toward 2030 World.

2. Global Health and Hypertension with Diversity (Racial, Economical Difference, Medical Resources).

3. Life-course and Hypertension (Preconception, Developmental Origins of Health and Disease (DOHaD), Cancer and hypertension).

4. Three Main Topics: Hypertension and Food, Move and Al.

5. Super-aged Society and Hypertension (Sarcopenia, Dementia, Social Capitals).

6. Hypertension Next Generation therapy (Renal Denervation, Single Compound Pills, Application).

7.Convergence of Communicable Diseases (Infectious diseases) and Non-Communicable Diseases (NCDs).

8. Japan Method for Conquering Hypertension (Hypertension Zero Town).

9. Pathophysiology of Hypertension: Chronicle to the Future.
10. Blood Pressure Measurement: Conventional and Future.

11. Imaging and Biomarker for Hypertension Management.

12. Hypertension Reigning Over Systemic Diseases.

13. Genetics, epigenetics, and microRNAs in blood pressure regulation.

We are also happy to announce the following important dates for the ISH2022 KYOTO.

Call for Abstracts: January 18 - April 19, 2022

Registration: Early Bird Registration will start in April 2022

We look forward to receiving abstracts from many of you.

The countdown for ISH2022 KYOTO has been stated on the official Twitter (@ish2022) as well as Instagram and Facebook. One year before the ISH2022 KYOTO, a countdown event was held at the Annual meeting of the Japanese Society of Hypertension in Okinawa, and the video of the event can be viewed on YouTube (Figure 5 ). 333 days before the ISH2022 KYOTO countdown was given by the ISH president, Prof. Maciej Tomaszewski, and other ISH core members will follow. Similarly, as we get closer to the ISH2022, we will be sending frequent countdown messages from the ISH members around the world. By sharing multidisciplinary wisdoms regarding hypertension and related diseases with each other, ISH2022 encourage the creation of novel paradigms for conquering hypertension. Through the participation to ISH2022 KYOTO on-site, we believe that participants will widen their knowledge, expertise and wisdom on hypertension by learning from the best lectures. We are looking forward to meeting you at KYOTO in 2022, and hope you enjoy the cultural and academic atmosphere of the city of Kyoto.

Please visit the official website for the updated information: https://www.ish2022.org/.

We are looking forward to meeting you in Kyoto in 2022.

Hiroshi Itoh (Keio University. President of ISH2022 KYOTO, Vice President of ISH, Former President of JSH) Kazutoshi Miyashita (Keio University. Secretary General of ISH2022 KYOTO) 
Figure 2. Cultural heritage sites and traditional sceneries in Kyoto. There are 17 different UNESCO World Heritage sites in the city, which make up one of the world's largest collections of temples and shrines.

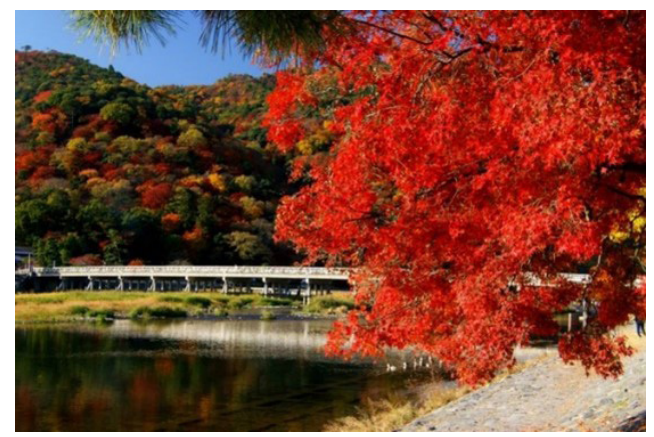

The Togetsu-kyo Bridge which has been a landmark in Arashiyama district for over four hundred years.

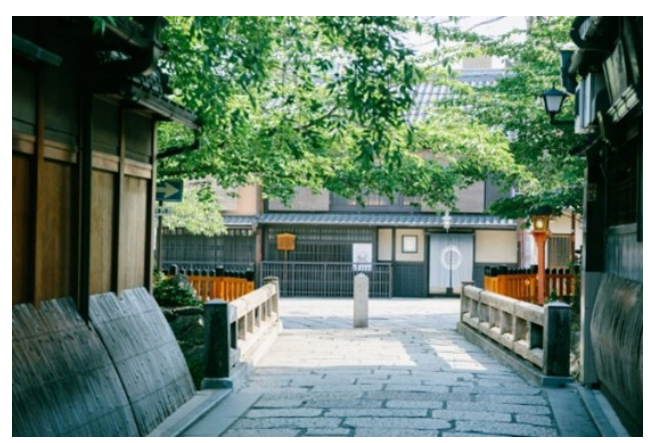

The Gion-Tatsumi Bridge which creates a photogenic scenery especially in the spring when the cherry blossoms are in full bloom.

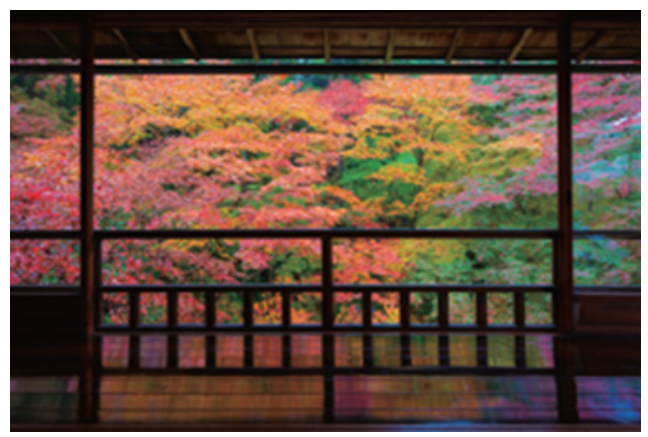

The Jissoin Temple in Iwakura district is famous for the superb garden scenery especially in the autumn.

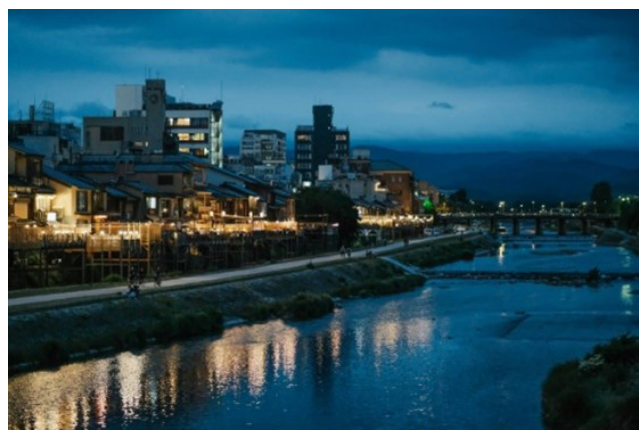

The Kamo River which is in the urban area of Kyoto city. Many citizens walk and rest in the riverside parks.

Figure 3. Special guests who have been scheduled to give lectures at the ISH2022 KYOTO:

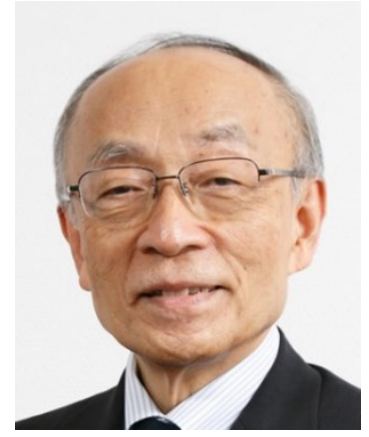

Dr. Hiroo Imura

President of the Japan Academy

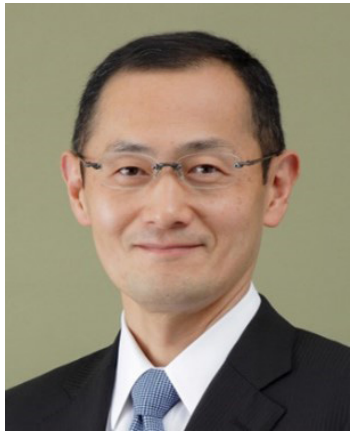

Dr. Shinya Yamanaka

Professor of Kyoto University, 2012 Laureate of Nobel Prize in Physiology or Medicine, who discovered induced pluripotent stem (iPS) cells

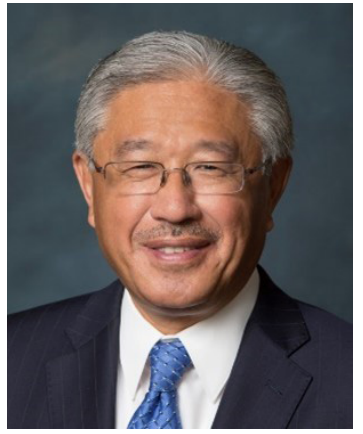

Dr. Victor J Dzau

President of the United States National Academy of Medicine

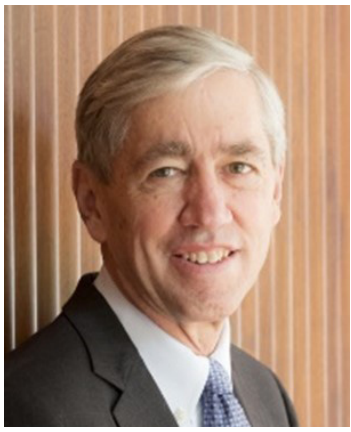

Dr. Richard P. Lifton

President of the Rockefeller University 
Figure 4. The three main topics of ISH2022 KYOTO scientific program: Food (food and nutrition), Move (exercise and fitness) and $\mathrm{Al}$ (artificial intelligence and digital health) that are related to hypertension.

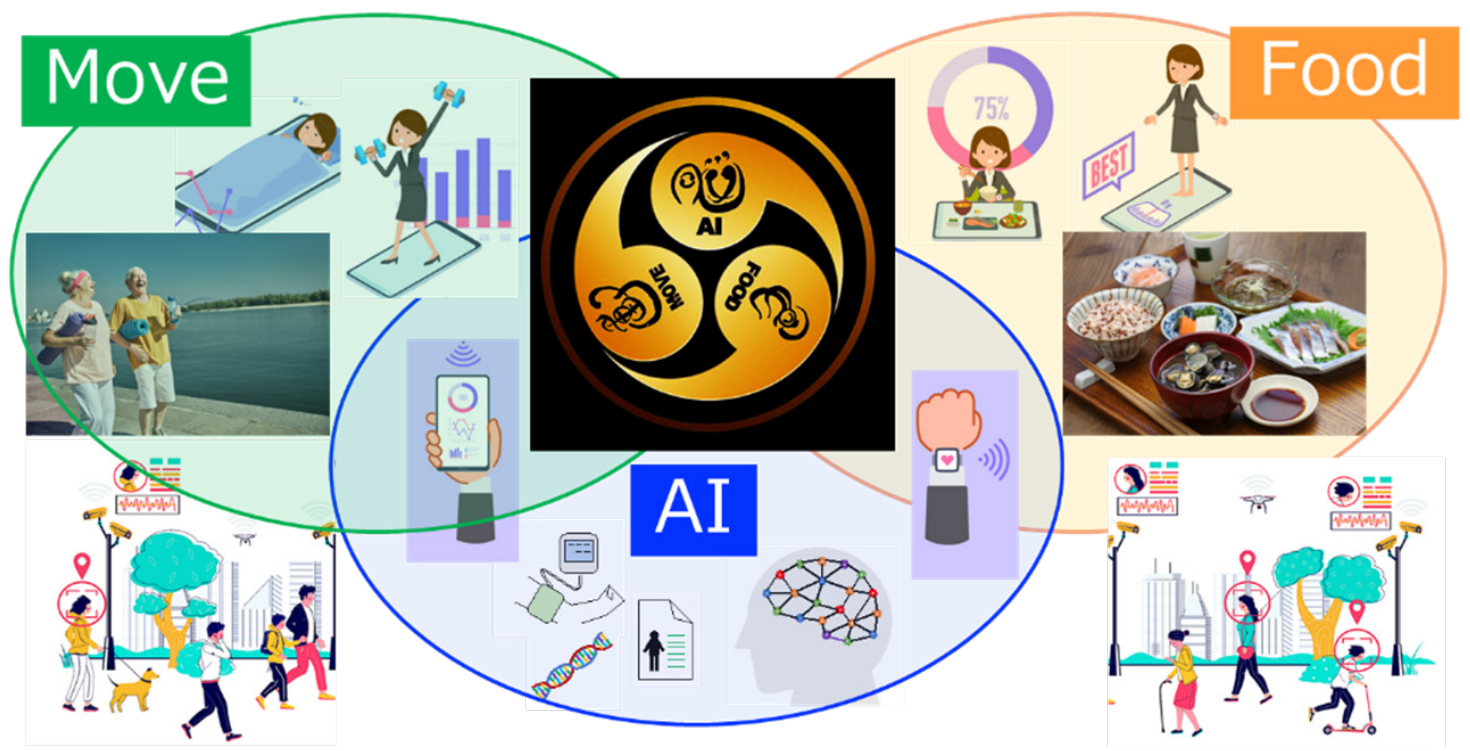

Figure 5. Countdown for ISH2022 KYOTO. The background is the Japan National Stadium which is the main stadium for 2020 TOKYO Olympic and Paralympic games.

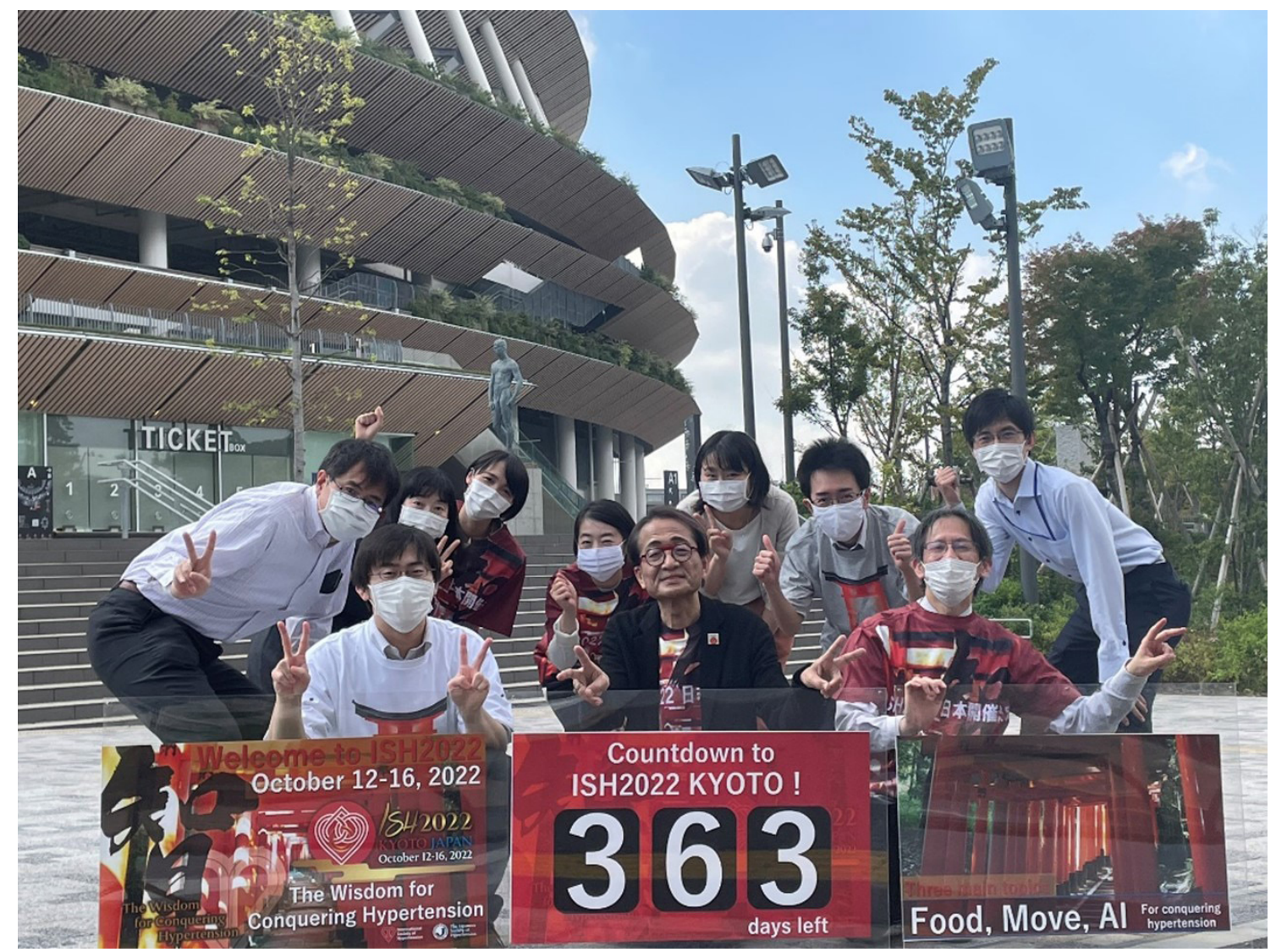

